\title{
COMPUTER SIMULATION OF THE UMER ELECTRON GUN*
}

\author{
I. Haber, Plasma Physics Division, Naval Research Laboratory, Washington, DC 20375
}

\author{
S. Bernal, R. A Kishek, P. G. O'Shea, M. Reiser, A. Valfells \\ Institute for Research in Electronics and Applied Physics, \\ University of Maryland, College Park MD 20742 \\ D. P. Grote, Heavy Ion Fusion - Virtual National Laboratory \\ Lawrence Berkeley National Laboratory, Berkeley, CA 94720
}

\begin{abstract}
An important feature of the UMER experimental program [1,2] is the continuing use of comparison between computer simulation and experimental measurement. These comparisons have, for example, explained several significant features observed in the prototype injector experiment. Therefore, during assembly and testing of the UMER injector two- and threedimensional simulations are being conducted with the aim of understanding the space-charge-dominated physics in the gun and injector transport sections, as well as interpreting experimental data. These simulations are important to providing a realistic initial beam distribution for simulating beam dynamics in the main ring. Emphasis here is on simulating the beam propagation in the gun region between the cathode grid and the anode grid for comparison with measurements at the exit from the gun structure.
\end{abstract}

\section{INTRODUCTION}

The University of Maryland Electron Ring (UMER)[1,2] has been designed to examine the physics of a highly space-charge-dominated electron beam as it propagates through hundreds of periods in an alternatinggradient transport system. Because this experimental apparatus can access a range of beam parameters not heretofore accessible, considerable effort has been expended in the careful design of a comprehensive set of beam diagnostics so that the beam can be accurately characterized and the physics clearly understood. Because of the complexity of the nonlinear physics expected to be of importance in a beam which is so highly space-charge-dominated, simulations have been a primary tool in designing the experiment and are expected to play a major role in interpreting the measured experimental data.
Many of the collective phenomena important to understanding the evolution of a space-charge-dominated beam have been found to depend on the detailed characteristics of the distribution function.[3] Excellent agreement was therefore obtained using the prototype injector for recent simulation-experiment comparisons.[4] The well-characterized initial distribution was created by passing the beam through an aperture that excluded all but the uniform central portion of the beam

Excellent agreement between simulation and experiment was also obtained in a previous[5] experiment where the beam was masked into five beamlet, which were then allowed to merge and form images of the original configuration. However, in this experiment, difficulty was encountered in attempts to incorporate an initially observed hollowing in the beam profile. This was attributed to inadequate knowledge of internal correlations in the beam distribution.

The possible presence of internal correlations, which are difficult to measure, can limit adequate prediction of the evolution of a space-charge-dominated beam. A simple example of this behavior would be the reconstruction of the distribution of an axisymmetric beam from $\mathrm{x}$ and $\mathrm{y}$ phase-space measurements. Though identical measurements in the two planes would strongly suggest axismmetry, there are other distributions that would also be consistent with the measured profile. Because of the importance of the initial distribution function to predicting beam evolution in UMER, it is important to begin simulations at the emitter surface, which is likely to be free of any significant internal correlations. "First principles" simulation of the beam starting from the cathode should then capture the beam dynamics, including any correlations that develop as the beam evolves.

\footnotetext{
* Work supported by the U. S. Department of Energy at NRL under contracts DE-AI02-93ER40799 and DE-AI02-94ER54232, at U. Md. under DE-FG02-92ER54178 and DE-FG02-94ER40855, and at the HIFVNL under DE-AC03-76SF00098 and W-7405-Eng-48.
} 


\section{SIMULATION METHODS}

Although it is desirable to conduct "first principles" simulations of the beam evolution beginning at the cathode surface, such a brute force simulation would be a very ambitious undertaking. A primary obstacle is the gun geometry, which has a grid $0.15 \mathrm{~mm}$ away from the cathode surface, and spacing of $0.15 \mathrm{~mm}$ between the grid wires. The complex geometry in the cathode-grid region can therefore substantially influence the characteristics of the emerging beam. Since this distance is much smaller than the cathode to anode distance, which is measured in centimeters, the disparate scales make it difficult to treat both regions accurately in a single simulation. Also complicating experiment/simulation comparisons is the difficulty of accurately measuring the grid potential and current when the beam is turned on because the grid floats at a $10 \mathrm{kV}$ potential.

It is also important to understand the time-dependent behavior of the gun structure, in order to simulate experiments on the longitudinal beam dynamics, since transients in the gun can be on the same time scale as the longitudinal phenomena to be measured. The simulations are therefore being performed using the combined plasma accelerator WARP code,[6] which is a fully self-consistent time dependent treatment. To make the runs tractable, the beam dynamics in the cathode-to-grid region are being treated separately from the cathode-to-anode region. The simulations discussed here have, to date, only examined the second region. The results should therefore be considered descriptive rather than predictive, since feedback between the two regions could change the detailed behavior.

A specified beam current and emittance is therefore injected into this region with a nominal 20 volt energy, which approximates the expected value for the actual grid-cathode voltage. However, the beam behavior has been found somewhat insensitive to the value of this voltage in the regime of gun operation. Parameters are then varied to understand the range of possible operating conditions.

To further reduce the numerical requirements, the threedimensional fields of the entire gun structure are solved and saved. The potential on a cylinder outside the beam is then applied as the boundary of a second simulation so that only the self-consistent field within this smaller region must be calculated. It was found that the added potential at the bounding surface from the presence of the beam is much less than 1 percent.

\section{SIMULATION RESULTS}

The gun configuration used in UMER, which employs a cathode grid to rapidly turn on the beam current, operates in a manner similar to the power amplification mode of a traditional vacuum triode.[7] Because of the need to understand details of the beam evolution as it is injected into the UMER ring, it is necessary to accurately characterize the beam phase space distribution. This is in contrast to merely describing the output current characteristics as has usually been done when describing operation of a vacuum triode.

One area of significance that will not be discussed here, is the modification to the beam characteristics that results from the geometric complexity in the region between the cathode and the cathode grid. Since the separation between the wires of the cathode grid is comparable to the distance from the emitting surface, both the longitudinal and transverse velocity distributions can be a complex function of the voltage applied to the grid structure. In view of the difficulty in measuring the actual grid to cathode potential, study of this behavior has been deferred pending a better understanding of the beam dynamics in the downstream region.

The simulations discussed here therefore assume that the beam is injected into the simulated region with a specified spatially uniform current and the idealized longitudinal and transverse velocities are assumed to be a Gaussian distribution emerging from the $\sim 0.1 \mathrm{eV}$ cathode. A cathode-grid to anode-grid distance of $220 \mathrm{~mm}$ was assumed as typical of gun operation. This distance can, in fact, be mechanically varied to adjust the gun perveance. With the latest upgrade to the pulser circuit, the gun was found to actually draw more than the nominally desired $138 \mathrm{~mA}$ which corresponds to the desired $120 \mathrm{~mA}$ nominal exit current at $10 \mathrm{kV}$, adjusted for the $87 \%$ transparency of the anode grid.

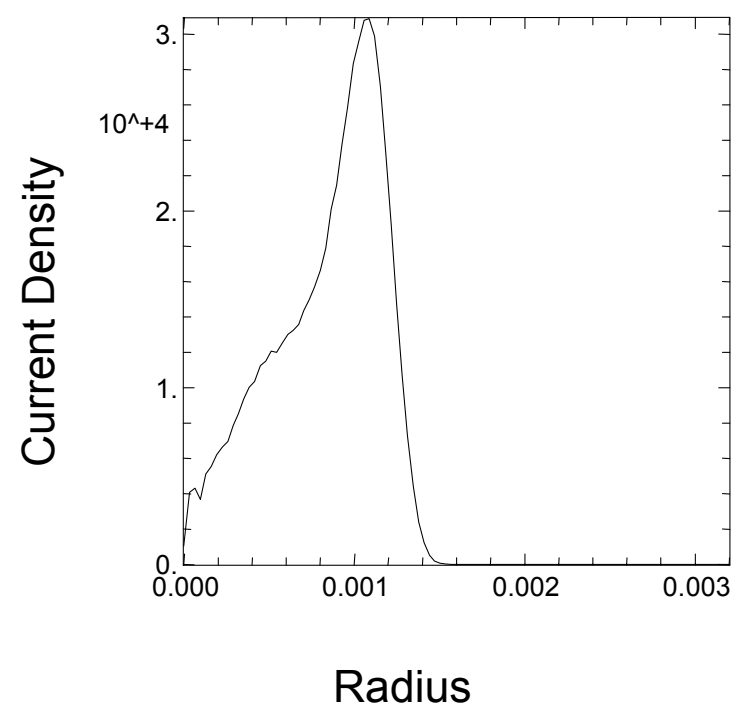

Fig. 1. Radial variation of the current density (in arbitrary units) at the plane of the anode grid, for an injected current of $138 \mathrm{~mA}$.

Figure 1 is a plot of the radial variation in current density for the nominal injected current of $138 \mathrm{~mA}$. At this current level, the beam is quite hollow. An interesting characteristic of the beam distribution is shown in Fig. 2, which is a plot of the rms thermal velocity as a function of radius. Because the orbits in the diode region are close to laminar, the truncation of a fluid moment expansion by neglecting the heat flow term can be used to predict a 
number of collective space-charge phenomena.[8] In the current case this approximation suggests that the beam in the diode region should approximately obey a local adiabatic approximation, so that the temperature as a function of radius should be proportional to the square root of the current density.

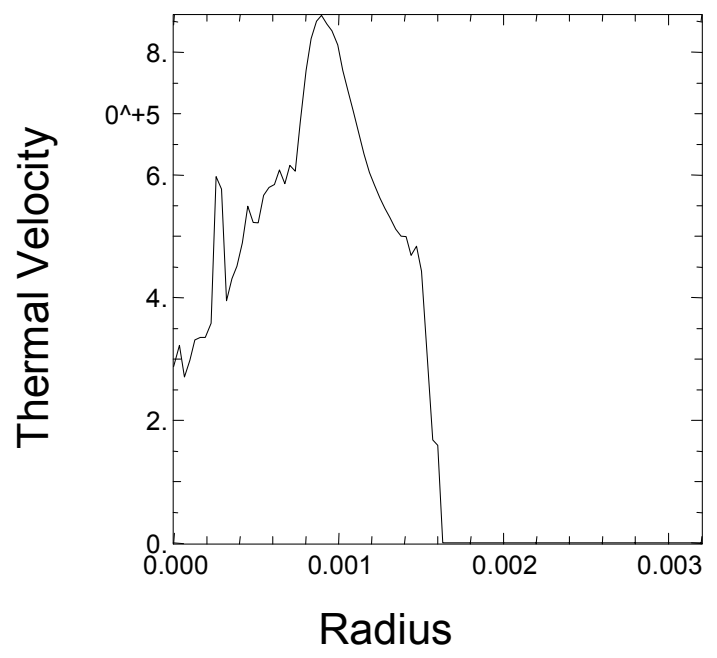

Fig. 2. Radial variation in rms velocity for the $138 \mathrm{~mA}$ beam plotted in Fig. 1.

The injected current was chosen with a degree of arbitrariness. Because the actually observed current in the diode under these conditions was somewhat greater than $138 \mathrm{~mA}$, a simulation was performed with the current increased by $50 \%$. The hollowing in current density across the beam is then somewhat reduced as shown in the plot in Fig. 3. Increasing the current to twice the initial run or $276 \mathrm{~mA}$ further decreases the observed hollowing so that the beam at the anode grid is almost uniform.

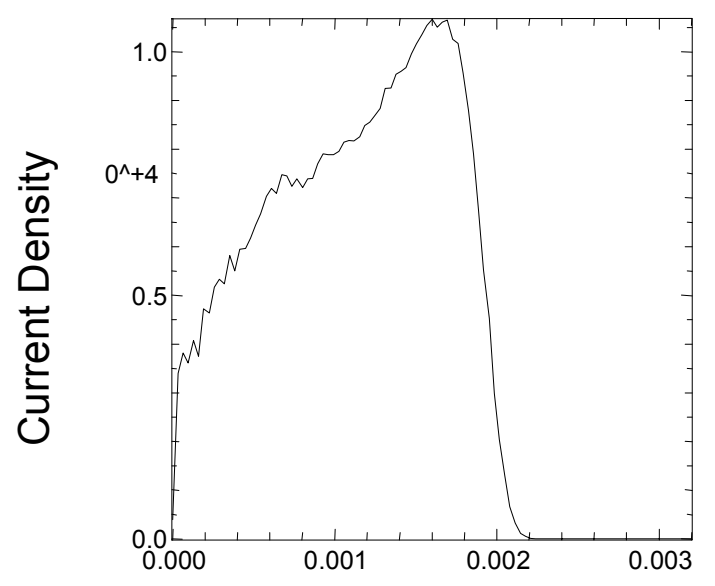

\section{Radius}

Fig 3. Radial variation in current density when the injected current is increased $50 \%$ to $207 \mathrm{~mA}$.
The simulations can also be used to examine the timedependent behavior of the beam. An interesting characteristic of the beam head is observed when the beam is turned on instantaneously at the cathode grid. The current reaching the anode still has an approximately 1 ns rise time which results from the central portion of the current pulse reaching the anode before the outer edges.

\section{CONCLUSIONS}

Preliminary simulations have been performed to examine beam characteristics in the region between the cathode grid and the anode grid in the UMER gun. The observed variability in the cross section as the current is varied should be useful, in concert with measurement, in characterizing details in the beam distribution important to predicting the downstream evolution, but difficult to obtain with simulation or measurement alone. Further work to characterize the beam behavior in the $0.15 \mathrm{~mm}$ region between the cathode and the cathode grid should assist in further refining this procedure and eventually approaching the ability to do "first-principles" predictions of the beam characteristics including the details of the three-dimensional phase space characteristics.

\section{ACKNOWLEDGEMENT}

The authors would like to thank David Kehne of FM technologies for his assistance.

\section{REFERENCES}

[1] P. O'Shea, et al. "The University of Maryland Electron Ring (UMER)," invited presentation at this conference.

[2] http://www.ireap.umd.edu/umer

[3] I. Haber, A. Friedman, D. P. Grote, S. M. Lund, R. A. Kishek, "Recent Progress in the Simulation of Heavy Ion Beams," Phys. Plasmas, 6, 2254 (May 1999).

[4] S. Bernal, R. A. Kishek, M. Reiser, and I. Haber, "Observations and simulations of Transverse Density Waves in a Collimated Space-Charge Dominated Electron Beam," Phys. Rev. Lett., 82, 20, 4002. (17 May 1999).

[5] I. Haber, D. Kehne, M. Reiser, and H. Rudd, "Experimental, Theoretical, and Numerical Investigation of the Homogenization of Density Nonuniformities in the Periodic Transport of a SpaceCharge Dominated Beam," Phys Rev A, 44, 5194 (Oct. 15, 1991).

[6] D. P. Grote, A. Friedman, I. Haber, W. Fawley, and J.L. Vay, New Developments in WARP: Progress Toward End-to-End Simulation," Nucl. Instr and Methods in Phys. Res. A 415 428-432 (1998).

[7] Karl. R. Spangenberg, Vacuum Tubes, (McGraw Hill, NY, 1948).

[8] Steven M. Lund, and Ronald C Davidson, "Warm Fluid Description of Intense Beam Equilibrium and electrostatic Stability Properties," Phys. Plasmas, 5, 3028 (Aug. 1998). 